\title{
Quantifying Preferences in Drug Benefit-Risk Decisions
}

Tommi Tervonen ${ }^{1 *}$, Aris Angelis ${ }^{2}$, Kimberley Hockley $^{3}$, Francesco Pignatti ${ }^{4}$, Lawrence D. Phillips ${ }^{5}$

${ }^{1}$ Evidera, London, UK; ${ }^{2}$ Department of Health Policy and LSE Health, London School of Economics and Political Science, UK; ${ }^{3}$ School of Public Health, Imperial College London, UK; ${ }^{4}$ European Medicines Agency, Amsterdam, The Netherlands; ${ }^{5}$ Department of Management, London School of Economics and Political Science, UK

\section{Conflict of Interest}

The authors declare that there is no conflict of interest.

\section{Funding Information}

This research has received no external funding.

\section{Keywords}

Pharmacoeconomics, public policy, quantitative methods, regulatory 


\begin{abstract}
Benefit-risk assessment in used in various phases along the drug lifecycle, such as marketing authorisation and surveillance, health technology assessment (HTA) and clinical decisions, to understand whether, and for which patients, a drug has a favourable or more valuable profile with reference to one or more comparators. Such assessments are inherently preference-based as several clinical and non-clinical outcomes of varying importance might act as evaluation criteria, and decision makers must establish acceptable trade-offs between these outcomes. Different healthcare stakeholder perspectives such as those of the patients and healthcare professionals are key for informing benefitrisk trade-offs. However, the degree to which such preferences inform the decision is often unclear as formal preference-based evaluation frameworks are generally not used for regulatory decisions, and if used, rarely communicated in HTA decisions. We argue that for better decisions, as well as for reasons of transparency, preferences in benefit-risk decisions should more often be quantified and communicated explicitly.
\end{abstract}

\title{
Introduction
}

The overall benefit-risk balance of any effective medicine is inherently a multi-dimensional concept consisting of assessing the balance of a drug's favourable effects or benefits against its unfavourable effects or risks (1). Any complete analysis of drug's benefit-risk balance must weigh these benefits against the risks (2), i.e., to incorporate preference information into the analysis. The weighting process should simultaneously consider the nature of the relevant benefit and risk outcomes- - their impact on the patient - as well as changes in likelihoods of the outcomes that are attributable to the treatment. Key decisions along the drug lifecycle, such as licensing, pricing and reimbursement, and treatment selection are commonly made by experts. However, it is seldom that preferences used in these decisions, i.e., acceptable trade-offs between the different benefit, risk and other decision criteria, are adequately quantified and communicated, if at all.

Different stakeholder groups sometimes exhibit different preferences, and within one group preferences may vary (3-6). Although not every decision made on drug benefit-risk balance needs 
detailed analysis to support it, this paper argues that decisions about drugs should more often include formal quantification of preferences. This is especially true for "preference-sensitive" decisions where multiple treatment options exist and there is no option that is clearly superior for all patients; or the evidence supporting one option over others is considerably uncertain or variable; or patients' views about the most important benefits and acceptable risks of a technology vary considerably within a population or differ from those of healthcare professionals (7). We have focused on the decisions relating to marketing authorization and health technology assessment (HTA), and clinical decisions. For each of these decision contexts, we have briefly reviewed the current state of practice in benefitrisk assessment and gaps on inclusion of preference information. Then, we have discussed practical recommendations for developing robust models that incorporate preference information.

\section{Current State of Practice in Benefit-Risk Assessment}

\section{Marketing Authorization}

Historically, European regulatory assessment has been justified mainly using implicit value judgments without a formal quantification of acceptable benefit-risk trade-offs (8). Appreciating the value of more quantitative approaches, European regulators called for a more explicit approach that includes decision criteria descriptions, data interpretation and valuations, and outcome weighting (9). The European Medicines Agency (EMA) has adopted a model that tabulates the magnitudes and uncertainties of the most important favourable (i.e. benefits) and unfavourable effects (i.e. risks) (10) in the 'effects table' (8). Construction of an effects table is one of the steps in developing a quantitative benefit-risk model with a framework such as PROACT-URL (11), belonging to the domain of Multi-Criteria Decision Analysis (MCDA). In the United States (US), the Food and Drug Administration (FDA) has adopted a Benefit-Risk Framework tool to communicate evidence and uncertainties about relevant benefits and risks taken into account in regulatory decisions (12). Decision making tools, including MCDA, have also been proposed for non-prescription drugs (13, 14). The International Council for Harmonization (ICH) allows for including preference information in marketing authorization applications in its 2016 update to the common technical document (15, 16). Despite EMA, FDA and ICH all proposing structured BRA guidance (8), they do not explicitly 
mandate the implementation of quantitative methodologies by applicant companies. Furthermore, the documentation of marketing authorization decisions generally consists of a qualitative description of the importance of the observed effects (clinical relevance) but does not include explicit preference information such as trade-offs, at least not in quantitative terms.

\section{Health Technology Assessment}

In the HTA context, payers typically focus on the metric of incremental cost-effectiveness ratio (ICER) of a new medical technology vs a comparator to reflect value-for-money considerations to guide their decision making and improve efficiency in resource allocation. For instance, as part of cost-utility analysis (CUA) adopted by prominent HTA agencies such as National Institute of Health and Care Excellence (NICE) in England and Tandvårds-läkemedelsförmånsverket (TLV) in Sweden, health effects are measured in quality-adjusted life years (QALY) with incremental cost per additional QALY gained acting as the efficiency metric (17). In England, the EuroQol EQ-5D instrument acts as the preferred measure of health-related quality of life (HRQoL) for the calculation of QALYs, usually using preference value sets that have been elicited from the general population (18). EQ-5D value set construction together with QALY calculation amounts to a process that is similar to benefit-risk assessment. However, preference elicitation methods commonly used in HTA (e.g., standard gamble and time trade-off exercises) are very different to those typically used in benefit-risk assessment (e.g., discrete choice experiments [DCE], MCDA swing weighting and thresholding exercises). Furthermore, the nature of the preference elicitation differs as the objective in HTA is to value permanent health states whereas benefit-risk assessments tend to value immediate clinical outcomes. HTA agencies implement ICER thresholds either implicitly or explicitly to reflect opportunity cost considerations, i.e., the benefit forgone that could have accrued from alternative coverage decisions. In practice, these thresholds are not derived using any evidence-based approach and empirical estimates of thresholds suggest that they are used inefficiently (19).

Due to the limited comprehensiveness of the QALY as a benefit component, payers often make decisions based on the parallel consideration of additional benefit dimensions from an evidence base that goes beyond "scientific value judgements" of clinical and economic evidence, to "societal value 
judgements" relating to disease severity, unmet medical need, and wider socioeconomic impact (2022). The consideration of these additional dimensions of value has traditionally been taking place in an ad hoc and implicit, if not informal, way. The use of multi-criteria evaluation methods has been proposed as an appropriate approach for developing more structured and transparent value frameworks that could overcome some limitations of economic evaluation techniques and achieve more comprehensive assessment (23-26). However, even following the development of good practices on the use of MCDA methods for $\operatorname{HTA}(27,28)$, the implementation of such approaches in practice has lagged (29). A possible explanation for this could be the number of methodological challenges pertinent to the use of MCDA in HTA, such as the need for non-additive modelling approaches and connection between criteria scales and weights $(30,31)$.

\section{Clinical Decisions}

Shared decision making between physician and patient occurs to some extent in clinical practice and has been suggested to improve care and reduce costs $(32,33)$. However, most medical decisions are still made by physicians with little input from patients due to various challenges (34). In theory, the same quantitative benefit-risk MCDA models that are usable for marketing authorization decisions are applicable for clinical decisions (35-37). Simple shared decision-making tools that capture patient input with visual-analogue or Likert scales may allow for more patient-centred treatment decisions (e.g., Hopkin and colleagues (38)). Clinical guidelines seem an appropriate place to include formal benefit-risk assessments and some guidelines currently explicitly discuss benefit-risk balance (e.g., Catapano and colleagues (39)). Including methodological experts into clinical guideline development may help increase the guidelines' impartiality (40). Formal benefit-risk assessments based on observed or expected treatment effects are already appearing in mainstream clinical journals (41-45), but they rarely incorporate preference information into the analysis beyond proxying clinical event importance, e.g., with mortality rates or health-state utility estimates (e.g., Dogliotti and Giugliano (46)). 


\section{Discussion}

The need for formal benefit-risk assessment of medicines is well recognized by decision makers and several structured approaches have been developed and currently used. However, the advantages of quantitative benefit-risk assessment have not been fully realized as preference data is rarely incorporated in a formal analysis. Preferences may differ between experts and between stakeholder groups. Patients may have different preferences to clinicians because of how the disease and its treatment affect their daily life beyond clinical outcomes. Experts often disagree because their professional experiences have been different. General practitioners, for example, see the world through different lenses than surgeons, and both build experiences that may lead to different preferences. Medical assessors have different risk attitudes as they "perceive the benefits and risks of medicines via a complex interplay of the medical situations, their personality traits and even their gender" (47). Quantifying and communicating such preferences in a benefit-risk model would enable decision makers to exchange views through rational discourse and test the effects of their judgements in the decision outcome, leading to better informed, more transparent decisions.

One reason for seemingly different preferences is that people exhibit different cognitive and motivational biases, which are well-documented by Kahneman (48). Although more than 150 biases have been found to date, Montibeller and von Winterfeldt (49) isolate just 12 cognitive and 14 motivational biases that apply in making decisions, and they provide suggestions for how these biases can be eliminated or reduced. Model builders should be aware of these biases and deploy debiasing techniques as appropriate.

Decision conferencing, where key stakeholders are brought together to develop value models by selecting evaluation criteria and eliciting preferences on these, is a usable format of model building that allows to control for biases (50). Decision conferencing has been successfully used in various contexts with different types of stakeholders (e.g., Angelis and colleagues, Nutt and colleagues, van Amsterdam and colleagues (51-53)). Benefit-risk models should be co-developed with groups of key stakeholders in workshops facilitated by an impartial specialist who guides the process but does not contribute to the content of the discussion. Indeed, the main purposes of decision conferencing are to 
achieve a shared understanding of the issues, a sense of common purpose, and commitment to a final model that best represents the consensus view of participants $(50,54)$. Decision conferencing is also a useful setting for developing attributes and agreeing on other aspects of the study protocol when preferences are elicited with a survey instrument such as the DCE.

Development of theoretically sound benefit-risk models is not easy and elicitation of stakeholder preferences for such models needs to account for critical study success factors, which are dependent on the chosen elicitation methodology (55). Preferences can be elicited efficiently with methods such as DCE (56), choice-based matching (57), threshold technique $(58,59)$, or swing weighting $(60)$, and the most appropriate method is dependent on characteristics of the benefit-risk assessment (55). Most elicitation methods result in preferences being measured on utility scales that can be difficult to interpret. Benefit-risk preferences should be communicated in terms of trade-offs that have a behavioural interpretation such as the maximum acceptable risk or the minimum acceptable benefit. Advanced analysis models allow quantifying preference heterogeneity in the target population (55), which is crucial for regulatory decisions and has been highlighted as critical study success factor by the FDA (7). However, although theoretical properties of most elicitation methods are wellunderstood, further research is warranted to understand better how method choice affects the results of preference elicitation.

Once constructed, the benefit-risk models can be used for re-assessment following new evidence generation (e.g., pharmacovigilance data or real-world evidence [RWE]) (9). Thus, the models should be developed with reusability in mind, ensuring that the elicitation is conducted with sufficiently wide performance ranges, to allow for evaluation of new evidence. Such benefit-risk models, if communicated transparently, may serve as reusable decision support tools in subsequent decisions. There is increased interest from both regulators and HTA agencies to formally incorporate patient preferences in benefit-risk analyses. NICE has worked in partnership with a patient organization to explore potential quantitative methods for capturing and using patient preferences within HTA (61). NICE has also provided scientific guidance for a patient preference study design (62). FDA's medical device regulation division has taken patient preferences into account in making regulatory decisions 
$(63,64)$. They have also released guidance suggesting that patients may provide useful experiential information, and that stated and revealed preference methods can be informative for understanding patient preferences (7). Similar guidance has been published by the public-private Medical Device Innovation Consortium (MDIC) (65). On the European side of drug regulation, EMA has conducted a study to investigate elicitation of patient preferences $(57,66)$ and is participating in the Innovative Medicines Initiative-Patient Preferences in Benefit-Risk Assessments during the Drug Life Cycle (IMI PREFER), a public-private partnership project that aims to shape the future of patient-centred benefit-risk assessment (67). These initiatives underline the importance of quantitative preferencebased benefit-risk assessments in drug decision making. However, solely the availability of such analyses on its own is insufficient; consideration of preferences in benefit-risk decisions is ultimately dependent on the decision makers' appreciation of the value of preference data. Training may be needed for decision makers who wish to be supported by quantitative benefit-risk models.

The traditional model of expert-led, agent-based health decision-making is evolving with the inclusion of patient input in various decisions of drug lifecycle, but key accountability and responsibility is still held by the decision makers. Thus, even though they make the final decision regardless of the context, we would expect that the decision rationale is communicated in a transparent manner. Understanding preferences that underlie regulatory benefit-risk decisions is key for informing subsequent decisions, which include clinical decisions. Decision makers have introduced a number of tools and frameworks for better communication of benefit-risk decisions. To build on this effort, we recommend quantifying and communicating preferences in drug benefit-risk decisions.

\section{Acknowledgements}

The authors acknowledge fruitful discussions on benefit-risk assessment leading to this paper with Shahrul Mt-Isa (Imperial College London and MSD Research Labs), Nikos Zafiropoulos (European Medicines Agency), Douwe Postmus and Hans Hillege (University Medical Center Groningen, The Netherlands). The authors also thank Emily Sargent (Evidera) and Vibha Shukla (Evidera) for 
editorial support, and two anonymous reviewers and associate editor for useful comments on earlier drafts of the manuscript.

\section{Author Contribution}

All authors contributed to writing of this manuscript.

\section{Disclaimer}

The views expressed in this article are the personal views of the authors and should not be understood or quoted as being made on behalf of, or reflecting the position of, the agencies or organizations with which the authors are affiliated.

\section{References}

(1) European Medicines Agency (EMA). Benefit-risk methodology. $<$ https://www.ema.europa.eu/en/about-us/support-research/benefit-risk-methodology> (2018). Accessed 12 December 2018.

(2) Hughes, D. et al. Recommendations for benefit-risk assessment methodologies and visual representations. Pharmacoepidemiol Drug Saf 25, 251-62 (2016).

(3) KCE. Incorporating Societal Preferences in Reimbursement Decisions: Relative Importance of Decision Criteria According to Belgian Citizens.

$<$ https://kce.fgov.be/sites/default/files/atoms/files/KCE_234_reimbursement_decisions_Repor t_0.pdf> (2014). Accessed 12 December 2018.

(4) Mol, P.G. et al. Understanding drug preferences, different perspectives. Br J Clin Pharmacol 79, 978-87 (2015).

(5) Muhlbacher, A.C. \& Juhnke, C. Patient preferences versus physicians' judgement: does it make a difference in healthcare decision making? Appl Health Econ Health Policy 11, 163 80 (2013).

(6) Muhlbacher, A.C. \& Nubling, M. Analysis of physicians' perspectives versus patients' preferences: direct assessment and discrete choice experiments in the therapy of multiple myeloma. Eur J Health Econ 12, 193-203 (2011).

(7) Food and Drug Administration (FDA). Patient Preference Information -Voluntary Submission, Review in Premarket Approval Applications, Humanitarian Device Exemption Applications, and De Novo Requests, and Inclusion in Decision Summaries and Device Labeling. Guidance for Industry, Food and Drug Administration Staff, and Other Stakeholders. $<$ https://www.fda.gov/downloads/medicaldevices/deviceregulationandguidance/guidancedocu ments/ucm446680.pdf> (24 August 2016). Accessed 12 December 2018.

(8) Pignatti, F. et al. Structured Frameworks to Increase the Transparency of the Assessment of Benefits and Risks of Medicines: Current Status and Possible Future Directions. Clin Pharmacol Ther 98, 522-33 (2015).

(9) Eichler, H.G., Abadie, E., Raine, J.M. \& Salmonson, T. Safe drugs and the cost of good intentions. N Engl J Med 360, 1378-80 (2009). 
(10) European Medicines Agency (EMA). Guidance document on the content of the critical assessment report. $<$ https://www.ema.europa.eu/documents/regulatory-proceduralguideline/day-80-assessment-report-quality-guidance-rev1217_en.pdf> (2016). Accessed 12 December 2018.

(11) Phillips, L. Is quantitative benefit-risk modelling of drugs desirable or possible? Drug Discov Today Technol 8, e1-e42 (2011).

(12) Food and Drug Administration (FDA). Benefit-Risk Assessment in Drug Regulartory Decision-Making. Draft PDUFA VI Implementation Plan (FY 2018-2022).

$<$ https://www.fda.gov/downloads/ForIndustry/UserFees/PrescriptionDrugUserFee/UCM6028 85.pdf> (30 March 2018). Accessed 12 December 2018.

(13) Brass, E.P., Lofstedt, R. \& Renn, O. Improving the Decision-Making Process for Nonprescription Drugs: A Framework for Benefit-Risk Assessment. Clin Pharmacol Ther 90, 791-803 (2011).

(14) Brass, E.P., Lofstedt, R. \& Renn, O. A Decision-Analysis Tool for Benefit-Risk Assessment of Nonprescription Drugs. The Journal of Clinical Pharmacology 53, 475-82 (2013).

(15) International Conference on Harmonization of Technical Requirements for Registration of Pharmaceuticals for Human Use (ICH). ICH Harmonised Treatment GUideline: Periodic Benefit-Risk Evaluation Report (PBRER) E2C9R2).

$<$ https://www.ich.org/fileadmin/Public_Web_Site/ICH_Products/Guidelines/Efficacy/E2C/E2 C R2 Step4.pdf $>$ (2012). Accessed 12 December 2018.

(16) International Conference on Harmonization of Technical Requirements for Registration of Pharmaceuticals for Human Use (ICH). Revision of M4E Guideline on Enhancing the Format and Structure of Benefit-risk Information in ICH: Efficacy - M4E(R2).

$<$ http://www.ich.org/fileadmin/Public_Web_Site/ICH_Products/CTD/M4E_R2_Efficacy/M4 E_R2 Step 4.pdf> (2016). Accessed 12 December 2018.

(17) National Institute for Health and Care Excellence (NICE). Process and Methods Guides. Developing NICE guidelines: the manual.

$<$ https://www.nice.org.uk/media/default/about/what-we-do/our-programmes/developing-niceguidelines-the-manual.pdf> (October 2014). Accessed 12 December 2018.

(18) Devlin, N.J., Shah, K.K., Feng, Y., Mulhern, B. \& van Hout, B. Valuing health-related quality of life: An EQ-5D-5L value set for England. Health Econ 27, 7-22 (2018).

(19) Claxton, K. et al. Methods for the Estimation of the NICE Cost Effectiveness Threshold. Health Technology Assessment 19, (2015).

(20) Angelis, A., Lange, A. \& Kanavos, P. Using health technology assessment to assess the value of new medicines: results of a systematic review and expert consultation across eight European countries. Eur J Health Econ 19, 123-52 (2018).

(21) de Folter, J., Trusheim, M., Jonsson, P. \& Garner, S. Decision-Components of Nice's Technology Appraisals Assessment Framework. Int J Technol Assess Health Care 34, 163 71 (2018).

(22) Nicod, E. \& Kanavos, P. Scientific and Social Value Judgments for Orphan Drugs in Health Technology Assessment. Int J Technol Assess Health Care 32, 218-32 (2016).

(23) Angelis, A. \& Kanavos, P. Multiple Criteria Decision Analysis (MCDA) for evaluating new medicines in Health Technology Assessment and beyond: The Advance Value Framework. Soc Sci Med 188, 137-56 (2017).

(24) EURORDIS Rare Diseases Europe. Mechanism of Coordinated Access to orphan medical products (MoCA). <https://www.eurordis.org/content/moca $>$ (2018). Accessed 12 December 2018.

(25) Goetghebeur, M.M., Wagner, M., Khoury, H., Levitt, R.J., Erickson, L.J. \& Rindress, D. Evidence and Value: Impact on DEcisionMaking--the EVIDEM framework and potential applications. BMC Health Serv Res 8, 270 (2008). 
(26) Institute for Clinical and Economic Review (ICER). ICER Value Assessment Framework. $<$ https://icer-review.org/methodology/icers-methods/icer-value-assessment-framework/>. Accessed 19 December 2018.

(27) Marsh, K. et al. Multiple Criteria Decision Analysis for Health Care Decision Making-Emerging Good Practices: Report 2 of the ISPOR MCDA Emerging Good Practices Task Force. Value Health 19, 125-37 (2016).

(28) Thokala, P. et al. Multiple Criteria Decision Analysis for Health Care Decision Making--An Introduction: Report 1 of the ISPOR MCDA Emerging Good Practices Task Force. Value Health 19, 1-13 (2016).

(29) Phillips, L.D. Best practice for MCDA in healthcare. In: Healthcare decisions supported by multi-currency decision analysis (eds. Baltussen, R., Marsh, K.D., Thokala, P. and Goetghebeur, M.M.) (Springer Publishing Company, New York, 2016).

(30) Marsh, K.D., Sculpher, M., Caro, J.J. \& Tervonen, T. The Use of MCDA in HTA: Great Potential, but More Effort Needed. Value Health 21, 394-7 (2018).

(31) Morton, A. Treacle and Smallpox: Two Tests for Multicriteria Decision Analysis Models in Health Technology Assessment. Value Health 20, 512-5 (2017).

(32) Oshima Lee, E. \& Emanuel, E.J. Shared decision making to improve care and reduce costs. $N$ Engl J Med 368, 6-8 (2013).

(33) Stiggelbout, A.M. et al. Shared decision making: really putting patients at the centre of healthcare. BMJ 344, e256 (2012).

(34) Hawley, S.T. Challenges to measuring and achieving shared decision-making in practice. Health Expect 21, 1-2 (2018).

(35) Mussen, F., Salek, S. \& Walker, S. A quantitative approach to benefit-risk assessment of medicines - part 1: the development of a new model using multi-criteria decision analysis. Pharmacoepidemiol Drug Saf 16 Suppl 1, S2-S15 (2007).

(36) Tervonen, T. et al. Applying Multiple Criteria Decision Analysis to Comparative BenefitRisk Assessment: Choosing among Statins in Primary Prevention. Med Decis Making 35, 859-71 (2015).

(37) Tervonen, T., van Valkenhoef, G., Buskens, E., Hillege, H.L. \& Postmus, D. A stochastic multicriteria model for evidence-based decision making in drug benefit-risk analysis. Stat Med 30, 1419-28 (2011).

(38) Hopkin, G., Au, A., Collier, V.J., Yudkin, J.S., Basu, S. \& Naci, H. Combining multiple treatment comparisons with personalized patient preferences: a randomized trial of an interactive platform for statin treatment selection. . Medical Decision Making, (2019).

(39) Catapano, A.L. et al. 2016 ESC/EAS Guidelines for the Management of Dyslipidaemias. Eur Heart J 37, 2999-3058 (2016).

(40) Ioannidis, J.P.A. Professional Societies Should Abstain From Authorship of Guidelines and Disease Definition Statements. Circ Cardiovasc Qual Outcomes 11, e004889 (2018).

(41) Cuzick, J. et al. Estimates of benefits and harms of prophylactic use of aspirin in the general population. Ann Oncol 26, 47-57 (2015).

(42) Pasea, L. et al. Personalising the decision for prolonged dual antiplatelet therapy: development, validation and potential impact of prognostic models for cardiovascular events and bleeding in myocardial infarction survivors. Eur Heart $J$ 38, 1048-55 (2017).

(43) Puhan, M.A., Yu, T., Stegeman, I., Varadhan, R., Singh, S. \& Boyd, C.M. Benefit-harm analysis and charts for individualized and preference-sensitive prevention: example of low dose aspirin for primary prevention of cardiovascular disease and cancer. BMC Med 13, 250 (2015).

(44) van Staa, T.P., Cooper, C., Barlow, D. \& Leufkens, H.G. Individualizing the risks and benefits of postmenopausal hormone therapy. Menopause 15, 374-81 (2008). 
(45) van Staa, T.P., Smeeth, L., Persson, I., Parkinson, J. \& Leufkens, H.G. What is the harmbenefit ratio of Cox-2 inhibitors? Int J Epidemiol 37, 405-13 (2008).

(46) Dogliotti, A. \& Giugliano, R.P. A novel approach indirectly comparing benefit-risk balance across anti-thrombotic therapies in patients with atrial fibrillation. Eur Heart J Cardiovasc Pharmacother 1, 15-28 (2015).

(47) Beyer, A.R., Fasolo, B., de Graeff, P.A. \& Hillege, H.L. Risk attitudes and personality traits predict perceptions of benefits and risks for medicinal products: a field study of European medical assessors. Value Health 18, 91-9 (2015).

(48) Kahneman, D. Thinking, Fast and Slow (Allen Lane: London, 2011).

(49) Montibeller, G. \& von Winterfeldt, D. Cognitive and Motivational Biases in Decision and Risk Analysis. Risk Anal 35, 1230-51 (2015).

(50) Phillips, L. Decision Conferencing. In: Advances in Decision Analysis: From Foundations to Applications (eds. Edwards, W., Miles, R.F. and von Winterfeldt, D.) (Cambridge University Press, Cambridge, 2007).

(51) Angelis, A., Montibeller, G., Hochhauser, D. \& Kanavos, P. Multiple criteria decision analysis in the context of health technology assessment: a simulation exercise on metastatic colorectal cancer with multiple stakeholders in the English setting. BMC Med Inform Decis Mak 17, 149 (2017).

(52) Nutt, D.J., King, L.A., Phillips, L.D. \& Independent Scientific Committee on, D. Drug harms in the UK: a multicriteria decision analysis. Lancet 376, 1558-65 (2010).

(53) van Amsterdam, J., Nutt, D., Phillips, L. \& van den Brink, W. European rating of drug harms. $J$ Psychopharmacol 29, 655-60 (2015).

(54) Franco, L.A. \& Montibeller, G. Facilitated modelling in operational research. Eur J Operation Res 205, 489-500 (2010).

(55) Tervonen, T. et al. MCDA swing weighting and discrete choice experiments for elicitation of patient benefit-risk preferences: a critical assessment. Pharmacoepidemiol Drug Saf 26, 1483-91 (2017).

(56) Lancsar, E. \& Louviere, J. Conducting discrete choice experiments to inform healthcare decision making: a user's guide. Pharmacoeconomics 26, 661-77 (2008).

(57) Postmus, D. et al. Individual Trade-Offs Between Possible Benefits and Risks of Cancer Treatments: Results from a Stated Preference Study with Patients with Multiple Myeloma. Oncologist 23, 44-51 (2018).

(58) Llewellyn-Thomas, H.A., Williams, J.I., Levy, L. \& Naylor, C.D. Using a trade-off technique to assess patients' treatment preferences for benign prostatic hyperplasia. Med Decis Making 16, 262-82 (1996).

(59) Brett Hauber, A., Fairchild, A.O. \& Reed Johnson, F. Quantifying benefit-risk preferences for medical interventions: an overview of a growing empirical literature. Appl Health Econ Health Policy 11, 319-29 (2013).

(60) Marsh, K. et al. Evaluation of COPD Treatments: A Multicriteria Decision Analysis of Aclidinium and Tiotropium in the United States. Value Health 20, 132-40 (2017).

(61) MyelomaUK. <https://www.myeloma.org.uk/news/survey-patient-preferences-in-healthtechnology-assessment-hta/> (Survey: patient preferences in Health Technology Assessment (HTA)). Accessed 12 December 2018.

(62) National Institute for Health and Care Excellence (NICE). NICE provides first scientific advice on patient preference study design. $<\mathrm{https} / /$ www.nice.org.uk/news/article/niceprovides-first-scientific-advice-on-patient-preference-study-design> (2019). Accessed March 142019.

(63) Ho, M.P. et al. Incorporating patient-preference evidence into regulatory decision making. Surg Endosc 29, 2984-93 (2015). 
(64) Department of Health \& Human Services. Traditional 510(k) Premarket Notification. K171331. . <https://www.accessdata.fda.gov/cdrh_docs/pdf17/k171331.pdf> (2017). Accessed 13 February 2019.

(65) Ho, M. et al. A Framework for Incorporating Patient Preferences Regarding Benefits and Risks into Regulatory Assessment of Medical Technologies. Value Health 19, 746-50 (2016).

(66) Postmus, D. et al. Incorporating patient preferences into drug development and regulatory decision making: Results from a quantitative pilot study with cancer patients, carers, and regulators. Clin Pharmacol Ther 99, 548-54 (2016).

(67) de Bekker-Grob, E.W. et al. Giving Patients' Preferences a Voice in Medical Treatment Life Cycle: The PREFER Public-Private Project. Patient 10, 263-6 (2017). 